\title{
Cannabis bei Krebs: Mehr Chancen als Risiken?
}

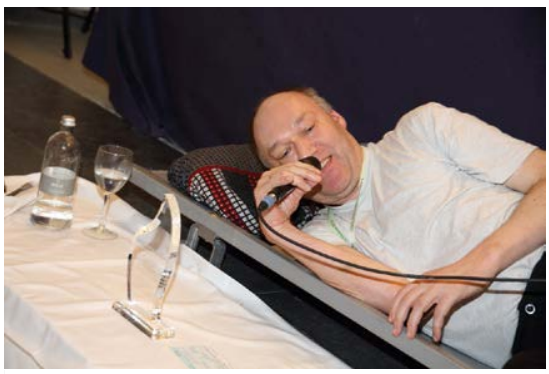

Dr. Franjo Grotenhermen während der Laudatio für den IACM Special Award, verliehen an Prof. Mahmoud Elsohly beim Kongress der IACM 2015. Foto: Autor

\section{Unser Gesprächspartner:}

Dr. med. Franjo Grotenhermen

Vorsitzender der Arbeitsgemeinschaft Cannabis als Medizin (ACM) und Geschäftsführer der International Association for Cannabinoid Medicines (IACM); Mitarbeiter am Kölner nova-Institut/ Abteilung nachwachsende Rohstoffe; eigene Praxis seit 2012.

DZO: Wie beurteilen Sie den Nutzen von Cannabis bei Krebserkrankungen? Welche wichtigen wissenschaftlichen Studien gibt es zu diesem Thema?

Man muss zwischen der symptomatischen Therapie von Appetitlosigkeit, Übelkeit, Schmerzen und Depressionen einerseits und krebshemmenden Eigenschaften von Cannabinoiden andererseits differenzieren. Die palliative Therapie von Krebserkrankungen mit cannabisbasierten Medikamenten ist aufgrund der klinischen Datenlage weitgehend akzeptiert. Anders sieht es mit dem Stellenwert von Cannabis zur Krebshemmung aus.

Im Internet kursiert eine Vielzahl von Erfahrungsberichten von Krebspatienten und Ärzten, die nahelegen, dass Cannabisprodukte in einzelnen Fällen das Überleben verlängern können. Allerdings ist oft unklar, ob die Krebserkrankungen wirklich aufgrund einer Therapie mit Cannabisprodukten verbessert wurden.
Die Behandlung mit Cannabis und seinen Wirkstoffen ist grundsätzlich eine mögliche komplementäre Option in der Krebstherapie. Wir wissen aus mehr als 100 tierexperimentellen Studien mit verschiedenen Cannabinoiden bei einer ganzen Reihe von Krebserkrankungen, dass Cannabinoide durch verschiedene Mechanismen krebshemmende Eigenschaften aufweisen. Im Labor zeigen sich insbesondere eine Hemmung des Krebswachstums, der Metastasierung, der Blutgefäßneubildung im Krebs, der Reduzierung der Resistenzbildung gegen bestimmte Chemotherapeutika sowie immunmodulatorische Effekte.

Im Tierversuch gab es allerdings auch drei Ausnahmen. Diese betreffen zwei Brustkrebs-Modelle mit Mäusen und ein Lungenkrebs-Modell, in denen THC das Wachstum der Tumoren förderte. Alle anderen Tierversuche bei unterschiedlichen Krebsarten, inklusive Brustkrebs und Lungenkrebs, ergaben, dass THC, CBD, CBG und viele synthetische Cannabinoide das Krebswachstum hemmen.

Epidemiologische und eine kontrollierte klinische Studie zeigen inzwischen, dass Cannabis bzw. dessen Cannabinoide nicht nur im Tierversuch, sondern auch bei Menschen krebshemmende Eigenschaften entfalten.

Verschiedene Institutionen in den USA von der Universität von Kalifornien und der Universität von Utah haben in Zusammenarbeit mit der Internationalen Agentur für Krebsforschung (IARC) der Weltgesundheitsorganisation die bisherigen epidemiologischen Daten zum Zusammenhang zwischen Cannabisrauchen und Krebs zusammengefasst. Danach gibt es bisher 34 epidemiologische Studien zu diesem Thema bei verschiedenen Krebsarten, insbesondere der Atemwege. Es gab zum Teil leicht erhöhte und zum Teil leicht erniedrigte Krebsrisiken durch das Rauchen von Cannabis. Die Autoren kamen zu dem Ergebnis, dass bisher keine abschließende Bewertung möglich ist und fordern weitere methodisch gute Studien zum Zusammenhang zwischen Cannabisrauchen und Krebs
[4]. Obwohl Cannabisrauch qualitativ die gleichen Verbrennungsprodukte wie Tabakrauch enthält, abgesehen von Nikotin beim Tabak und Cannabinoiden beim Cannabis, fiel in der größten epidemiologischen Studie auf [3], dass Cannabisraucher ein statistisch nicht signifikant um etwa 20-30\% erniedrigtes Risiko für Lungenkrebs hatten, während Tabakrauchen mit einer erheblichen Zunahme des Risikos verbunden war.

Erstmals wurden im Jahr 2017 vorläufige Ergebnisse einer Studie veröffentlicht [7], die zeigte, dass Cannabis nicht nur im Tierversuch, sondern auch beim Menschen das Krebswachstum hemmen und das Überleben verbessern könnte. An dieser Studie nahmen 21 Patienten mit einem Rezidiv eines Glioblastoms teil. 12 Patienten erhielten das Standard-Chemotherapeutikum Temozolomid plus Cannabisextrakt in Form eines Mundsprays, 9 Patienten erhielten die Standardtherapie mit Temozolomid plus Placebo. Nach einem Jahr lebten noch 53\% der Patienten, die nur Temozolomid und das Plazebo erhalten hatten, während die 1-Jahresüberlebensrate in der CannabisGruppe $83 \%$ betrug. Diese Ergebnisse sprechen dafür, dass der Zusatz von Cannabinoiden das Therapieergebnis von Standardtherapien möglicherweise verbessern kann. Bisher gibt es jedoch nur eine Pressemitteilung des Cannabisextrakt-Herstellers und noch keine Publikation in einer wissenschaftlichen Zeitschrift.

Im Frühjahr 2018 wurde eine Studie aus Israel veröffentlicht [1]. Etwa 3000 Krebspatienten waren zwischen 2015 und 2017 mit Cannabis behandelt worden. Das Ergebnis zeigt, dass Cannabis viele Symptome lindern kann, die bei einer Krebserkrankung auftreten können, darunter Schlafstörungen, Übelkeit, Appetitlosigkeit, Schwächegefühl und Schmerzen.

Auf die Studie will ich etwas konkreter eingehen: Nach 6 Monaten waren 902 Patienten gestorben und 682 hatten die Behandlung abgebrochen. Von den übrigen knapp 1500 Patienten beantworteten 1211 (60,6\%) die Fragen der Forscher. Bei 95,9\% hatte sich der 
Zustand durch Cannabis verbessert, $45 \mathrm{~Pa}$ tienten (3,7\%) gaben keine Änderung an. 4 Patienten (0,3\%) schrieben dem Cannabis eine Verschlechterung ihres medizinischen Zustands zu. Die häufigsten Symptome zu Beginn der Beobachtung waren Schlafstörungen (78\%), Schmerzen (78\%), Schwäche (73\%), Übelkeit (65\%) und mangelnder Appetit (49\%). Die Autoren folgerten, dass Cannabis „als eine palliative Behandlung von Krebspatienten eine gut verträgliche, wirksame und sichere Option darstellt, um Menschen zu helfen, durch bösartige Erkrankungen verursachte Symptome zu bewältigen“.

Es entspricht auch meinen persönlichen Erfahrungen, dass viele Krebspatienten von einer Cannabistherapie profitieren. Sie dürfen darauf hoffen, dass ihre Symptome nachlassen. Wesentlich zurückhaltender in der Beurteilung von Cannabis bzw. einzelner Cannabinoide muss man sein, wenn es um klinisch relevante krebshemmende Eigenschaften geht.

DZO: Welche Patienten profitieren besonders von einer Gabe?

Schätzungen zufolge profitieren 10-20\% der Schmerzpatienten trotz aller Bemühungen von einer alleinigen konventionellen Schmerztherapie nicht, so dass hier ein Therapieversuch mit Cannabinoiden sinnvoll wäre. In der Krebstherapie ist Cannabis ein Baustein unter vielen und besonders bei Patienten mit fortgeschrittenen Tumorstadien geeignet, um zum Beispiel Schmerzen oder Übelkeit zu lindern oder einer Gewichtsabnahme entgegenzuwirken.

DZO: Welche praktischen Erfahrungen haben Sie bei Krebserkrankungen gemacht?

Man muss grundsätzlich zwei Dinge auseinanderhalten, die oft vermischt oder ungenau diskutiert werden. Zum einen ist uns kein zweites Molekül auf der Erde bekannt, das ein derart breites therapeutisches Potenzial besitzt, wie das THC der Cannabispflanze: Es lindert Schmerzen und regt den Appetit an, hemmt Entzündungen und löst Muskelkrämpfe, bekämpft Übelkeit und senkt einen erhöhten Augeninnendruck, Albträume bei Patienten mit posttraumatischer Belastungsstörung werden reduziert und die Bronchien von Asthmatikern erweitert etc.
Andererseits sind die Wirkungen bei vielen Patienten mit solchen Erkrankungen nur gering oder moderat. Bei Schmerzen beispielsweise hilft Cannabis etwa jedem dritten oder vierten Patienten ausreichend gut bei guter Verträglichkeit. Auch bei vielen anderen möglichen Einsatzgebieten profitiert im Allgemeinen nur ein Teil der Betroffenen. Im Internet liest man vor allem Berichte von Patienten, denen Cannabis gut geholfen hat, und weniger von jenen, bei denen es nicht oder nur gering wirksam war. Solche Erfahrungsberichte verzerren daher den Blick auf die Realität.

Bei Patienten, die auf Cannabis-Medikamente ansprechen, ist die Freude natürlich groß. Dank guter Verträglichkeit können sie die Arzneien über Jahrzehnte verwenden. Sie brauchen keine Angst vor Nebenwirkungen haben, die ihre inneren Organe wie Magen, Leber, Nieren oder Herz schädigen.

Ein positives Beispiel zur krebshemmenden Wirkung aus meiner Praxistätigkeit: Im Juli 2016 besuchte mich ein 74-Jahre alter Mann in meiner Praxis. Er litt an wiederkehrenden Basalzellkarzinomen sowie an einer aktinischen Keratose. Basaliome auf der Nase wurden bei meinem Patienten erstmals im Jahr 2003 diagnostiziert und 2004 und 2014 mit Operation bzw. einer Bestrahlung behandelt. Im Jahr 2006 wurde eine großflächige Hauttransplantation auf die Nase vorgenommen. Im Februar 2016 war das Basaliom an zwei Stellen auf der Nase zurückgekehrt. In Absprache mit seinem Hautarzt hatte er eine kleine Menge eines THC-reichen Cannabisextrakts (Haschischöl) viermal täglich auf die betroffenen Stellen appliziert. Innerhalb von zwei Wochen heilten sie vollständig ab.

DZO: Worin unterscheiden sich die verschiedenen Präparate? Ist es wichtig, bei Krebserkrankungen beide Cannabinoide, also sowohl das psychotrope Delta-9-Tetrahydrocannabinol (THC) als auch das nicht psychotrope Cannabidiol (CBD) zu verabreichen?

Während für THC schmerzlindernde, appetitsteigernde und muskelentspannende Eigenschaften im Vordergrund stehen, wirkt CBD vor allem angstlösend und entzündungshemmend. THC und CBD hemmen das Tumorwachstum, nur in wenigen Aus- nahmefällen wirkte THC in tierexperimentellen Untersuchungen tumorfördernd. Die Wirkungen von CBD und THC sind dosisabhängig. Viel THC ist wirksamer als wenig THC. Eine Kombination von THC und CBD erwies sich in den bisher durchgeführten experimentellen Untersuchungen wirksamer als jedes der beiden Cannabinoide allein. Es ist bisher aber nicht bekannt, wie das optimale Verhältnis von THC zu CBD aussehen sollte. Es gibt Hinweise, dass das Verhältnis bei verschiedenen Krebserkrankungen unterschiedlich sein könnte. So war beispielsweise CBD in Tierexperimenten zum Brustkrebs wirksamer als THC. Da CBD gut vertragen wird, kann dieses Cannabinoid in hohen Dosen eingenommen werden. Wird CBD gut vertragen, so sollte zusätzlich THC in einer möglichst hohen Dosis eingenommen werden.

DZO: Welche Applikationsformen sind denkbar?

Grundsätzlich kommt es für die Wirksamkeit nicht auf die Art und Weise der Einnahme an, sondern auf die Menge von THC und $\mathrm{CBD}$, die in die Blutbahn und damit zum Tumor gelangt. So ist es ein im Internet weit verbreiteter Irrtum, dass Extrakte mit höheren THC-Gehalten oder höheren CBDGehalten wirksamer seien als Extrakte mit geringeren Cannabinoid-Konzentrationen. Nur bei topischer Applikation, wie etwa beim Basaliom, sollte ein hoch konzentrierter Extrakt verwendet werden.

Es ist auch nicht entscheidend, ob die Cannabinoide oral eingenommen oder ob sie inhaliert werden. Eine rektale Verabreichung von Cannabinoiden ist weniger wirksam, da die Cannabinoide über die Schleimhaut des Enddarms schlecht aufgenommen werden.

Es gibt fertige Extrakte, wie vor allem den Cannabisextrakt Sativex, der THC und CBD zu gleichen Teilen enthält. 3 Fläschchen, die insgesamt $810 \mathrm{mg}$ THC und $750 \mathrm{mg}$ CBD enthalten, kosten $314,14 €$. Reines Dronabinol (THC) und reines Cannabidiol (CBD) aus der Apotheke sind teurer, wenn man sie einzeln erwirbt.

Außerdem ist es nicht kompliziert, selbst einfache Extrakte aus Cannabisblüten herzustellen. Diese sind günstiger als im Internet erhältliche Haschischöle (RSO-Öl, Can- 
nabisöl, etc.) sowie in der Apotheke erhältliche Extrakte wie Sativex. Bei der Wahl der Blüten kann man sich am THC-Gehalt orientieren. So kann man die Sorten mit 20\% oder mehr THC verwenden, um das meiste THC für das Geld zu bekommen.

Wichtig ist, dass Cannabinoide vor der Einnahme decarboxyliert werden. Dazu können Cannabisblüten vor der Einnahme erhitzt werden, beispielsweise bei $140^{\circ} \mathrm{C}$ für etwa 15 Minuten oder bei $110^{\circ} \mathrm{C}$ für etwa 45 Minuten. Dies kann durch Erhitzen in einem Öl geschehen, aber auch beispielsweise durch Erhitzen im Backofen, die bevorzugte Methode meiner Patienten. Bei $100{ }^{\circ} \mathrm{C}$ werden mindestens 60 Minuten zur vollständigen Decarboxylierung benötigt, sodass eine Teezubereitung, bei der Cannabisblüten nur einige Minuten auf $100^{\circ} \mathrm{Cer}$ hitzt werden, lediglich eine geringe Ausbeute ermöglicht. Eine Teezubereitung ist aber möglich, wenn die Cannabinoide in den Blüten zuvor bei höheren Temperaturen decarboxyliert wurden.

Grundsätzlich kann die Decarboxylierung nach diesem Schema auch in einem Trockenschrank einer Apotheke erfolgen. Um eine cannabistypische Geruchentwicklung in der Apotheke zu vermeiden, sollte das Cannabis in einem geeigneten Gefäß erhitzt werden, dass erst nach Abkühlung wieder geöffnet wird.

DZO: Welche Nebenwirkungen können bei einer Therapie mit Cannabis auftreten?

Akute Nebenwirkungen sind bei einer Behandlung mit Cannabinoiden oder Cannabis recht häufig. Sie betreffen vor allem die Psyche und die psychomotorische Leistungsfähigkeit sowie das Herz-Kreislauf-System. Häufige Nebenwirkungen sind Müdigkeit, Schwindelgefühl, Blutdruckabfall, Herzrasen und Mundtrockenheit. Bei regelmäßiger Einnahme tritt meist eine Gewöhnung ein. Entzugserscheinungen und Schädigungen der geistigen Fähigkeiten wurden nur bei sehr hohen Dosierungen beobachtet.

Bei einer Therapie mit cannabisbasierten Medikamenten ist es wichtig, mit kleinen Mengen zu beginnen und die Dosis dann ganz langsam zu steigern, damit keine starken Nebenwirkungen auftreten. Bei jedem Patienten muss die individuell wirksame und verträgliche Dosierung ermittelt werden. Das dauert meistens ein bis zwei Wochen, manchmal auch etwas länger.

DZO: Welche Kontraindikationen müssen berücksichtigt werden?

Cannabis soll bei schweren Herz-KreislaufErkrankungen, Psychosen sowie bei Schwangeren und stillenden Müttern nicht angewandt werden. Die Behandlung von Kindern und Jugendlichen vor der Pubertät sollte sehr sorgfältig abgewogen werden. Pharmakokinetische Wechselwirkungen sind mit einer Vielzahl von Arzneimitteln möglich, die auch über die Leber bzw. das Cytochrom-P450-System ab- und umgebaut werden. Pharmakodynamische Wechselwirkungen betreffen vor allem sedierende Substanzen (Alkohol, Benzodiazepine) sowie Medikamente, die die Herzfrequenz beeinflussen (Betablocker, Amphetamine).

DZO: Gibt es spezifische Wechselwirkungen mit Medikamenten in der Onkologie?

Von Wechselwirkungen sind vor allem Tamoxifen und Immuntherapien betroffen. Ein Metabolit von Tamoxifen ist ein inverser Agonist am CB2-Rezeptor (Cannabinoid2-Rezeptor), sodass unerwünschte Wechselwirkungen auftreten könnten [2]. Ich rate daher bei einer Behandlung mit Tamoxifen von einer komplementären Behandlung mit Cannabinoiden ab. CBD und THC besitzen immunsuppressive Eigenschaften, die ihre Wirksamkeit bei Entzündungen und Allergien erklären. Solche Eigenschaften sind bei vielen Immuntherapien nicht erwünscht, sodass auch hier Vorsicht geboten ist.

THC und CBD hemmen transmembranäre Arzneimitteltransportsysteme. Dazu gehören beispielsweise das P-Glykoprotein ( $\mathrm{P}$ $g p)$, das Breast Cancer Resistance Protein (BCRP) oder das Multidrug Resistance-Related Protein 1 (MRP1) [5]. Transportproteine schleusen fremde Stoffe wie Zytostatika (Substrate) aus einer Zelle heraus und bestimmen damit wesentlich die Wirksamkeit einer Tumortherapie. Werden die Transportproteine in ihrer Aktivität gehemmt, kann beispielsweise die Bioverfügbarkeit der entsprechenden Krebsmedikamente steigen. Die klinische Relevanz dieser möglichen Wechselwirkungen auf die Toxizität der Zytostatika lässt sich bisher nicht beur- teilen. Da die Bioverfügbarkeit betroffener Zytostatika durch die hemmende Wirkung der Cannabinoide steigt, wird deren Wirksamkeit möglicherweise gesteigert, sodass zunächst keine Abschwächung der Standardtherapie erwartet werden kann.

THC und CBD werden in der Leber über Enzyme der Cytochrom-P450-Familie - darunter CYP2C9 und CYP3A4 - verstoffwechselt. Deshalb können Wechselwirkungen mit Medikamenten auftreten, die auf dem gleichen Wege metabolisiert werden. Substrate von CYP3A1 sind beispielsweise Cyclophosphamid, Paclitaxel und Tyrosinkinasehemmer (Imatinib und andere). Cyclophosphamid ist zusätzlich ein Substrat von CYP2C9. Die klinische Relevanz dieser Wechselwirkungen ist bisher nicht bekannt.

DZO: Wie sieht es aus mit der Verkehrstüchtigkeit?

Die Einnahme von THC-haltigen Cannabisprodukten kann die psychomotorische Leistungsfähigkeit und damit die Fahrtüchtigkeit beeinträchtigen. Nach wissenschaftlichen Erkenntnissen ist die Fähigkeit zur aktiven Teilnahme am Straßenverkehr jedoch nur während des akuten Cannabisrausches, der beim Rauchen von Cannabisprodukten etwa 2-4 Stunden anhält, reduziert und führt nicht zu einer anhaltenden Ungeeignetheit zum Führen von Kraftfahrzeugen.

Rechtlich sieht das deutsche Straßenverkehrsgesetz (StVG) seit 1998 die Möglichkeit vor, das Führen eines Kraftfahrzeuges nicht nur unter dem Einfluss von Alkohol, sondern auch unter dem Einfluss anderer berauschender Mittel als Ordnungswidrigkeit zu behandeln und mit einer Geldbuße zu bestrafen.

Nach § 24a Absatz 2 des StVG gilt: „Ordnungswidrig handelt, wer unter der Wirkung eines in der Anlage zu dieser Vorschrift genannten berauschenden Mittels im Straßenverkehr ein Kraftfahrzeug führt. Eine solche Wirkung liegt vor, wenn eine in dieser Anlage genannte Substanz im Blut nachgewiesen wird. Satz 1 gilt nicht, wenn die Substanz aus der bestimmungsgemäßen Einnahme eines für einen konkreten Krankheitsfall verschriebenen Arzneimittels herrührt.“ Der Gesetzgeber erlaubt daher die Teilnahme am Straßenverkehr, 
wenn Patienten von einem Arzt Dronabinol, Nabilon (Canemes ${ }^{\circledR}$ ), Sativex ${ }^{\circledR}$ oder Cannabisblüten verschrieben wurde.

Ich schreibe in meine Dosierungsanleitungen grundsätzlich folgenden Satz: „In der Dosierungsphase sollte die Teilnahme am Straßenverkehr vermieden werden, bis Sie sich wieder sicher fühlen. “ [6]

Nach der Fahrerlaubnisverordnung schließt regelmäßiger Cannabiskonsum die Teilnahme am Straßenverkehr aus, sodass der Führerschein von Cannabiskonsumenten eingezogen wird.

Die Bundesopiumstelle und das Bundesverkehrsministerium werten die medizinische Verwendung von Cannabisblüten straßenverkehrsrechtlich wie die Verwendung von Dronabinol, Nabilon oder Sativex ${ }^{\circledR}$, sodass die betroffenen Patienten grundsätzlich ihren Führerschein behalten können. Eine Führerscheinstelle kann jedoch auch bei der medizinischen Verwendung von Medikamenten eine Überprüfung der Fahreignung durch ein ärztliches Gutachten oder eine MPU (Medizinisch-Psychologische Untersuchung) veranlassen, wenn Zweifel an der Fahreignung auftreten.

DZO: Für welche Indikationen darf Cannabis bei Krebspatienten verordnet werden?

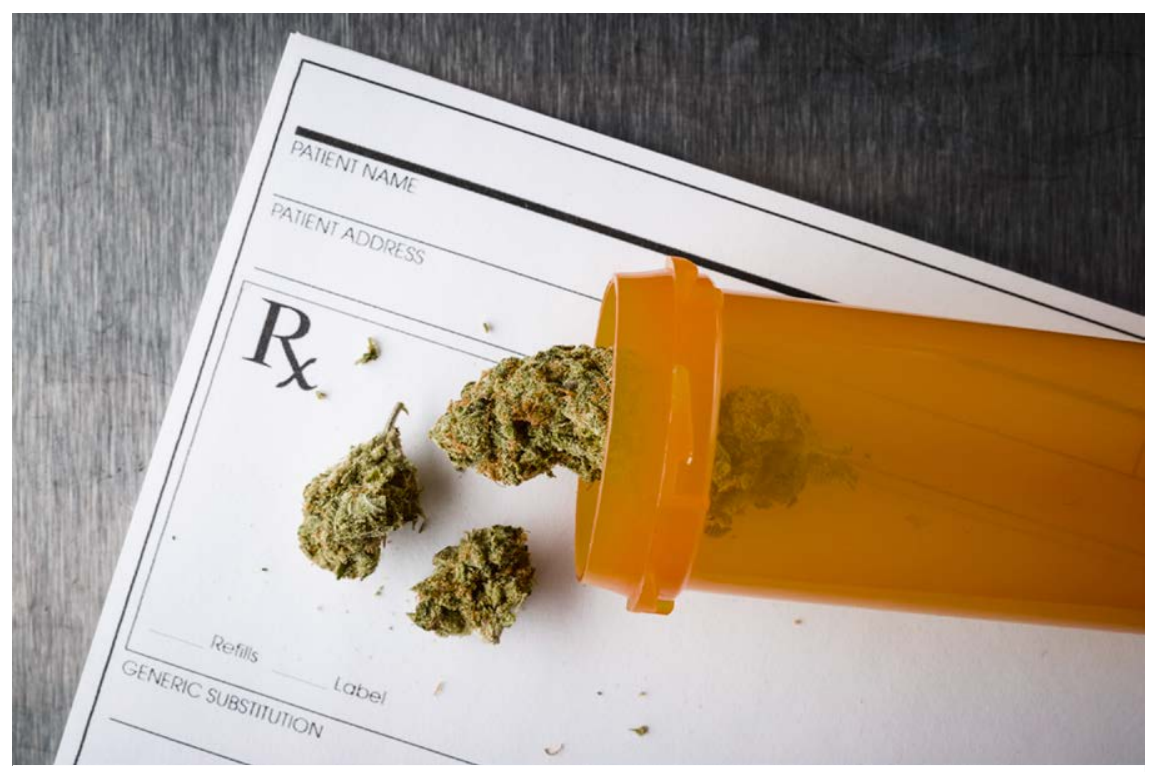

„Viele Ärzte sind zurückhaltend, weil sie die Bürokratie, Belastungen des Budgets und mögliche Regresse wegen unwirtschaftlichen Handelns fürchten." ( $)$ Adobe Stock/goodmanphoto
Durch ein Gesetz, das im März 2017 in Kraft getreten ist, können Schwerkranke mittels Betäubungsmittelrezept Cannabisblüten und Extrakte in der Apotheke erhalten. Damit wurde es möglich, deutlich mehr Patienten mit den benötigten Präparaten zu behandeln.

Das Gesetz schreibt nicht vor, bei welchen Indikationen Cannabis eingesetzt werden darf, sondern hier gilt wie für andere Betäubungsmittel der § 13 BtMG, der die Anwendung von Betäubungsmitteln auf Patienten einschränkt, die mit anderen Mitteln nicht behandelt werden können.

DZO: Welche Voraussetzungen müssen erfüllt sein, damit die Kosten von den Krankenkassen übernommen werden?

Die Kosten dürfen von den Krankenkassen nach § 31 Abs. 6 SGB V „nur in begründeten Ausnahmefällen“ abgelehnt werden. Die Krankenkassen müssen die Kosten erstatten, wenn eine schwere Erkrankung vorliegt, und wenn „eine allgemein anerkannte, dem medizinischen Standard entsprechende Leistung im Einzelfall nicht zur Verfügung steht“ oder wenn diese Leistung „im Einzelfall nach der begründeten Einschätzung des behandelnden Vertragsarztes unter Abwägung der zu erwartenden Nebenwirkungen und unter Berücksichtigung des Krankheitszustandes der oder des
Versicherten nicht zur Anwendung kommen kann“.

Zudem muss „eine nicht ganz entfernt liegende Aussicht auf eine spürbare positive Einwirkung auf den Krankheitsverlauf oder auf schwerwiegende Symptome“ bestehen. Dieser Punkt trifft für eine Krebstherapie mit Cannabis grundsätzlich nicht zu. Anders sieht es bei der palliativen Verwendung THC-haltiger Präparate, etwa zur Behandlung der Appetitlosigkeit mit Kachexie, einer therapieresistenten Übelkeit oder opiatresistenten Schmerzen aus.

Allerdings müssen Patienten vor der Einlösung eines Rezeptes bei den Krankenkassen einen Antrag auf Kostenübernahme stellen und den Bescheid abwarten. Eine Therapie oder ein Therapiebeginn auf einem Privatrezept ist dagegen jederzeit möglich.

DZO: Welche besonderen Pflichten muss der verordnende Arzt beachten?

Bei der Ausstellung eines Privatrezeptes muss der Arzt keine besonderen Pflichten beachten. Bei einer Verordnung zulasten der gesetzlichen Krankenkassen muss er für jede Cannabis-Behandlung Daten anonym an das Bundesinstitut für Arzneimittel und Medizinprodukte senden (www.begleiterhebung.de). Sind der Arzt oder der Patient dazu nicht bereit, werden die Kosten von den Krankenkassen nicht erstattet.

DZO: Welche Herausforderungen sehen Sie für die Zukunft?

Gegenwärtig sehen wir, dass sich die Krankenkassen häufig aus der Verantwortung stehlen. So wird zunehmend infrage gestellt, ob eine Erkrankung schwerwiegend ist, ob Standardtherapien ausgeschöpft wurden oder eine Aussicht auf eine spürbare Einwirkung auf den Krankheitsverlauf besteht. Letzteres trifft insbesondere zu, wenn die klinische Datenlage unbefriedigend ist. Kürzlich hat das Bundesverfassungsgericht das Urteil eines Landessozialgerichts bestätigt, nach dem die Krankenkasse einem Patienten mit Cluster-Kopfschmerzen die Kosten nicht erstatten muss, da es bisher keine ausreichenden klinischen Daten gibt. Das führt zu der von Ärzten und betroffenen Patienten schwer akzeptierbaren Situation, dass ein schwerkranker Patient, der für seine Umwelt 
und seinen Arzt offensichtlich von einer Therapie mit Cannabis profitiert, die Krankenkasse aber aufgrund der Datenlage eine Kostenübernahme ablehnen kann, weil es keine begründete Aussicht auf Linderung gäbe.

Ich habe den Eindruck, dass diese Einschränkung der ärztlichen Therapiefreiheit nicht dem Wunsch des Gesetzgebers entsprach, als der Bundestag das Gesetz am 19. Januar 2017 einstimmig und ohne Enthaltungen verabschiedete. Viele Ärzte sind zudem zurückhaltend, weil sie die Bürokratie (Kostenübernahmeantrag, Meldung an die Bundesopiumstelle), Belastungen des Arzneimittelbudgets und mögliche Regresse wegen unwirtschaftlichen Handelns fürchten.

Wir arbeiten daher weiter daran, dass die Therapieentscheidung von Ärztinnen und Ärzten aufgrund ihrer medizinischen Einschätzung getroffen werden kann und darf und nicht (zu sehr) durch medizinfremde Einflüsse bestimmt wird. Die Diskussion über den Sinn und Unsinn der Verwendung von Cannabis-Medikamenten bei bestimmten Indikationen sollte innerhalb der Ärzteschaft und nicht zwischen einzelnen Ärzten und Krankenkassen geführt werden.

DZO: Herr Dr. Grotenhermen, zum Schluss noch eine persönliche Frage: Was tun Sie für sich, um gesund zu bleiben?

Da ich seit nunmehr etwa 30 Jahren chronisch erkrankt und eigentlich seit 1992 berentet bin, versuche ich mein Leben so zu gestalten, dass ich mit dieser Erkrankung möglichst lange gut leben kann. Das impliziert neben spezifischen Behandlungsverfahren alle Lebensbereiche, inklusive Ernährung, Genussmittel, Arbeit und Bewegung. So verwende ich mit Ausnahme meiner Medikamente keine Drogen (Koffein, Nikotin, Alkohol, etc.) und esse im Allgemeinen nur einmal am Tag (mittags).

\section{HILFREICHE LINKS}

www.cannabis-med.org

www.arbeitsgemeinschaft-cannabis-

medizin.de

Bundesopiumstelle: www.bfarm.de

\section{Weiterführende Literatur}

Grotenhermen F. Cannabis gegen Krebs. Solothurn: Nachtschatten Verlag; 2017. Buchbesprechung s. S. 203

\section{Literatur}

[1] Bar-Lev Schleider L, Mechoulam R, Lederman $V$ et al. Prospective analysis of safety and efficacy of medical cannabis in large unselected population of patients with cancer. Eur J Intern Med 2018; 49: 37-43

[2] Franks LN, Ford BM, Fujiwara T et al. The tamoxifen derivative ridaifen- $B$ is a high affinity selective $C B 2$ receptor inverse agonist exhibiting anti-inflammatory and anti-osteoclastogenic effects. Toxicol Appl Pharmacol 2018; 353: 31-42
[3] Hashibe M, Morgenstern H, Cui Y et al. Marijuana use and the risk of lung and upper aerodigestive tract cancers: results of a population-based case-control study. Cancer Epidemiol Biomarkers Prev 2006: 15: 1829-1834

[4] Hashibe M, Straif K, Tashkin DP et al. Epidemiologic review of marijuana use and cancer risk. Alcohol 2005; 35: 265-275

[5] Iffland K, Grotenhermen F. An update on safety and side effects of cannabidiol: a review of clinical data and relevant animal studies. Cannabis Cannabinoid Res 2017; 2: 139-154

[6] Musterdosierungsanleitungen für Dronabinol, Sativex, Nabilon und Cannabisblüten http://www.cannabis-med. org/german/dosierungsanleitung.rtf

[7] Pressemitteilung von GW Pharmaceuticals vom 7. Februar 2017. Verfügbar online unter http://www.gwpharm.com/about-us/ news/gw-pharmaceuticals-achieves-positive-results-phase-2-proof-concept-studyglioma

\section{Korrespondenzadresse}

Dr. med. Franjo Grotenhermen

Am Mildenweg 6

59602 Rüthen

praxis@dr-grotenhermen.de

www.dr-grotenhermen.de

\section{Bibliografie}

DOI https://doi.org/10.1055/a-0758-8908

Deutsche Zeitschrift für Onkologie

2018; 50: 188-192

(c) Georg Thieme Verlag KG Stuttgart · New York ISSN 1617-5891 